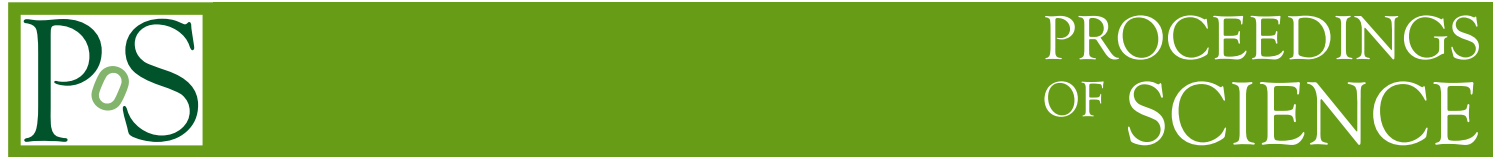

\title{
Jet results from the Tevatron
}

\section{Christophe Royon*}

IRFU-SPP, CEA Saclay, F91 191 Gif-sur-Yvette cedex, France

E-mail: christophe.royon@cea.fr

On behalf of the CDF and DO Collaborations

We describe the most recent results on jet physics from the D0 and CDF collaborations, starting from jet inclusive cross sections and $\alpha_{S}$ measurements and moving to dijet and three-jet cross section measurements.

35th International Conference of High Energy Physics - ICHEP2010,

July 22-28, 2010

Paris France

${ }^{*}$ Speaker. 


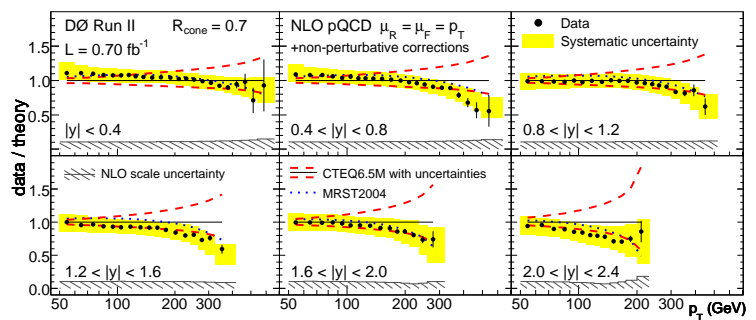

Figure 1: Data over theory for the inclusive $p_{T}$ cross section measurement for the D0 collaboration using the 0.7 jet cone.

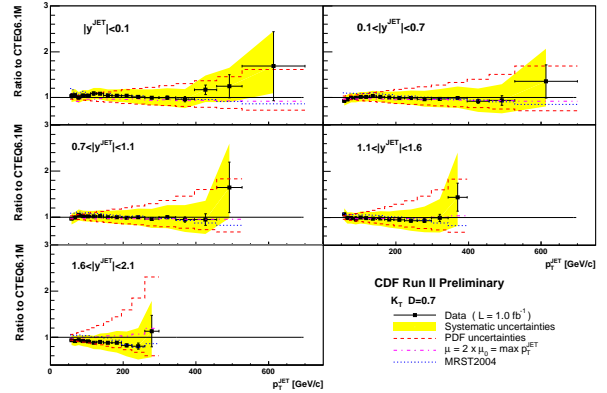

Figure 2: Data over theory for the inclusive $p_{T}$ cross section measurement for the CDF collaboration using the $k_{T}$ algorithm.

In this report, we discuss the jet physics results and perspectives at the Tevatron and the LHC. HERA allows to reach very low values of $x$ at low $Q^{2}\left(x \sim 10^{-6}\right)$, whereas the Tevatron and the LHC reach very high values of $Q^{2}$ at high $x\left(Q^{2} \sim 3.10^{5}\right.$ and $10^{8} \mathrm{GeV}^{2}$ at the Tevatron and the LHC respectively), allowing to probe QCD at very high energies.

\section{Inclusive jet cross section measurements}

The inclusive jet cross section measurements at the Tevatron and the LHC rely on the determination of the jet energy calibration, which leads to the largest systematic uncertainties. The jet energy scale is determined mainly using $\gamma+$ jet events. In the D0 collaboration, the corrected jet energy is obtained using the following method $E_{\text {jet }}^{c o r r}=\frac{E_{\text {jet }}^{\text {uncorr }}-O f f}{\text { Show } \times \text { Resp }}$ where $E_{\text {jet }}^{c o r r}$ and $E_{\text {jet }}^{\text {uncorr }}$ are the corrected and uncorrected jet energies respectively. The offset corrections $(O f f)$ are related to uranium noise and pile-up and are determined using zero-bias data. The showering corrections (Show) take into account the energy emitted outside the jet cone because of the detector and dead material. The jet response (Resp) is the largest correction, and can be subdivided in few corrections: the equalization of the calorimeter response as a function of rapidity, and measurement of the jet response for the central part of the calorimeter only using the $p_{T}$ balance in $\gamma+$ jet events. Some additional small corrections related to the method biases are introduced. One important additional correction deals with the difference in response between quark and gluon jets. The difference was studied both in data and in Monte Carlo (using for instance the $\gamma+$ jet and the dijet samples which are respectively quark and gluon dominated) and leads to a difference of 4 to $6 \%$ as a function of jet $p_{T}$. The jet energy scale is thus not universal but sample dependent. QCD jets (gluon dominated) will have a different correction with respect to the $t \bar{t}$ events for instance which are quark dominated. The uncertainties reached by the D0 collaboration concerning the determination of jet energy scale are of the order of $1.2 \%$ for jet $p_{T}$ between $70-400 \mathrm{GeV}$ and in a wide range of rapidity around zero. The CDF collaboration follows a method which is more Monte Carlo oriented using beam tests and single pion response to tune their Monte Carlo.

The measurement of the inclusive jet cross section [1] was performed using a jet cone algorithm with a cone size of 0.7 (D0 and CDF) and the $k_{T}$ algorithm (CDF). Data are corrected to 


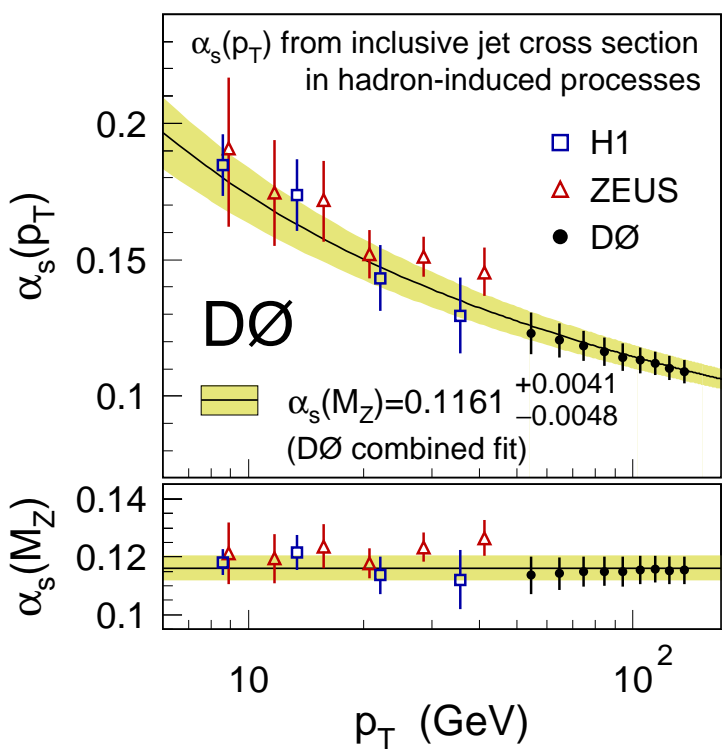

Figure 3: $\alpha_{S}$ measurement from the D0 collaboration compared to the HERA results at lower $p_{T}$.

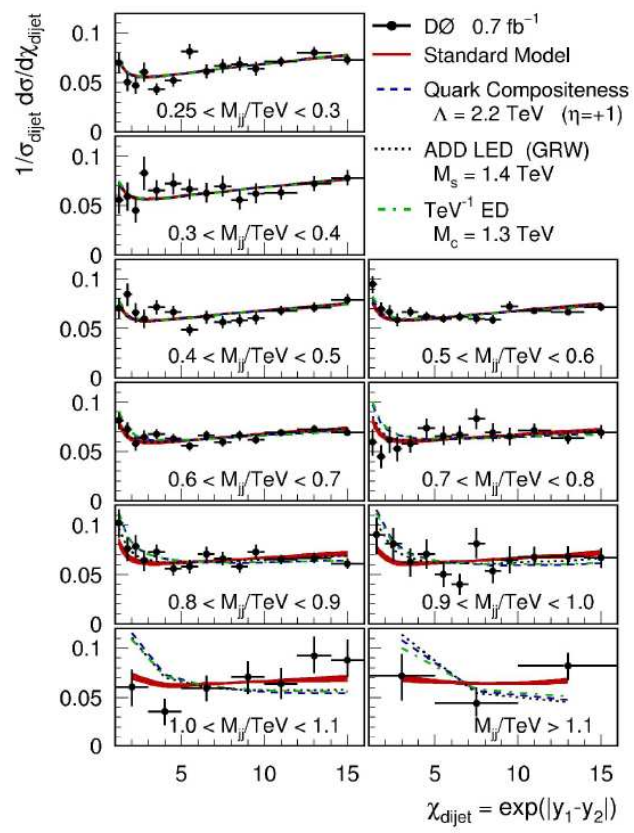

Figure 4: Dijet angular distributions measured by the D0 experiment in 10 regions of dijet mass above $250 \mathrm{GeV}$. No excess was found with respect to QCD which leads to limits on quark compositeness at about 3 $\mathrm{TeV}$.

hadron level (D0 and CDF cone jets) or parton level (CDF $k_{T}$ jets). Data are compared with NLO QCD calculations using either CTEQ6.5M [2] for D0 or CTEQ6.1 [2] for CDF (the uncertainties of the CTEQ6.5M parametrisation are two times smaller). The ratio data over theory is in good agreement with NLO QCD calculation (see Figs. 1 and 2) with a tendency of the CTEQ parametrisation to be slightly lower than the data at high jet $p_{T}$. The MRST2004 [2] parametrisation follows the shape of the measurements. The uncertainties are lower than the PDF ones and will allow to constrain further the PDFs. The D0 collaboration took also special care of the uncertainty correlation studies, by giving the effects of the 24 sources of systematics in data [1].

\section{2. $\alpha_{S}$ measurement in the D0 collaboration}

The inclusive jet cross section is directly related to the measurement of $\alpha_{S}, \sigma_{\text {pert }}\left(\alpha_{S}\right)=$ $\left(\Sigma_{n} \alpha_{S}^{n} c_{n}\right) \otimes f_{1}\left(\alpha_{S}\right) \otimes f_{2}\left(\alpha_{S}\right)$ where the $c_{n}$ coefficients are known at NLO and the $f_{1}$ and $f_{2}$ proton parton density dependence on $\alpha_{S}$ are known from the PDFs. The renormalisation group equations allow to know $\alpha_{S}$ at any scale from $\alpha_{S}$ at the $Z$ boson mass. The chosen PDF is MSTW 2008 [3] since it is given for 21 values of $\alpha_{S}\left(M_{Z}\right)$ in the range $0.11-0.13$. Since the inclusive cross section measurements are mainly used to measure PDFs, it is needed to minimize the correlations between the data and the PDFs, and it is necessary to restrict this analysis to the kinematic regions where the Tevatron data do not dominate in the PDF determination. Only 21 data points of the inclu- 
sive cross section are kept for the $\alpha_{S}$ determination. This measurement leads to the most precise determination of $\alpha_{S}$ from an hadronic collider [4] $\alpha_{S}\left(M_{Z}\right)=0.1161_{-0.0041}^{+0.0048}$ and is shown in Fig. 3 .

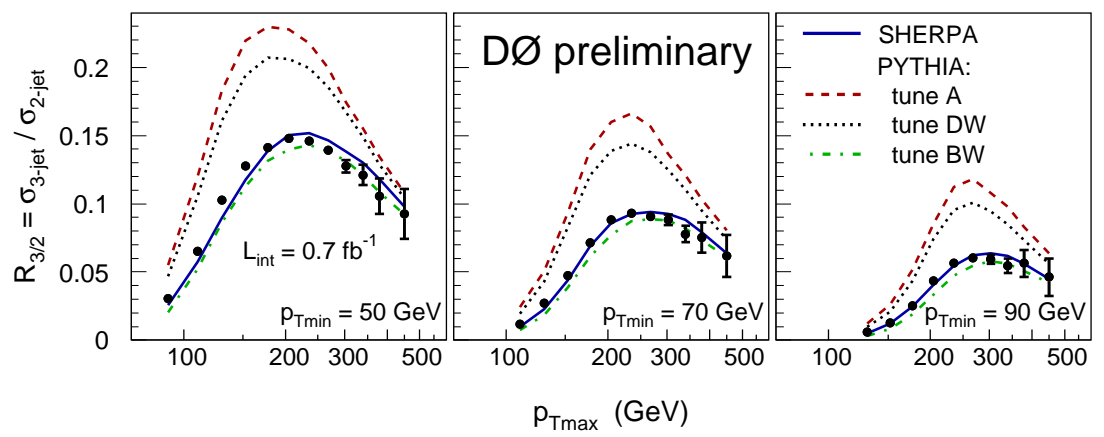

Figure 5: Measurement of the 3 jet to 2 jet cross section ratios by the D0 collaboration. A good agreement is found with the Sherpa generator while the pythia generator show a too large ratio except for the BW tune.

\section{Dijet cross section and $\chi$ measurements}

In addition, the D0 collaboration measured the dijet mass cross section in six rapidity bins, and data and QCD predictions are in good agreement at medium rapidities whereas data are lower than the QCD predictions at high rapidity [5]. The CDF collaboration measured the dijet mass cross section [6] above $180 \mathrm{GeV}$, and up to $1.3 \mathrm{TeV}$. No excess was found with respect to NLO QCD calculations and this measurement allows to exclude excited quarks below $870 \mathrm{GeV}$, axigluon below $1.25 \mathrm{TeV}$ and technirho below $1.1 \mathrm{TeV}$.

The D0 collaboration measured in addition the $\chi=\exp \left(\left|y_{1}-y_{2}\right|\right)$ variable sensitive to new physics in ten different regions of dijet mass above $250 \mathrm{GeV}$ [7] as shown in Fig. 4. New physics which might appear appear at low $\chi$ values in the highest dijet mass bins. No excess was found with respect to perturbative QCD, this measurement leads to the best limits on quark compositeness at $3 \mathrm{TeV}$ and extra-dimensions at $1.3-1.9 \mathrm{TeV}$. The D0 collaboration also measured the 3 jet mass and a reasonable agreement between data and NLO QCD prediction was found [8].

\section{Measurement of multi-jet cross section}

The D0 collaboration performed the first measurement of the ratios of multijet cross sections at the Tevatron. This allows to probe QCD more independently of PDFs (there is a residual dependence only due to the difference in quark and gluon subprocess composition) and to probe $\alpha_{S}$ in a direct way. In Fig 5, we display the ratio of the 3 to 2-jet cross sections, and a good agreement is found with Sherpa 1.1.3 [9] while almost all Pythia [10] tunes lead to a too high ratio [11].

\section{Measurement of the mass distribution of high $p_{T}$ jets}

The CDF collaboration measured recently the mass of the high $p_{T}$ jets [12]. Such a measurement is motivated since high mass jets form a significant background to searches for new physics, 


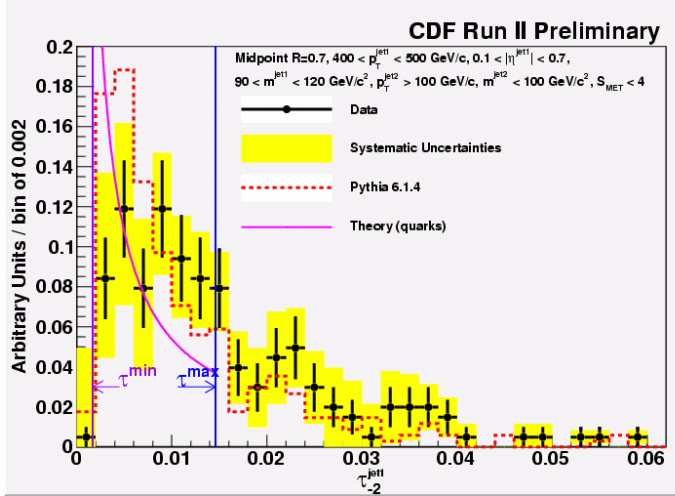

Figure 6: Measurement of the angularity for high $p_{T}$ jet events by the CDF collaboration.

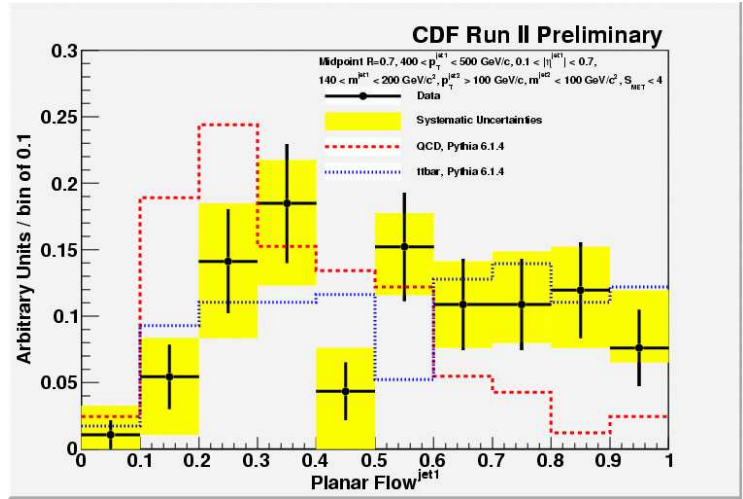

Figure 7: Measurement of the planar flow for high $p_{T}$ jet events by the CDF collaboration.

for the Higgs bosons and for the top quark jets at high $p_{T}$. Angular and planar flow variables are used since they allow to test QCD easily and they are robust against soft radiation and less dependent on the jet algorithm. A good agreement is found between data and QCD predictions over the mass range between 70 to $250 \mathrm{GeV}$ and data interpolate betwen quark and gluon showering. The angularity measures the energy distribution inside a jet and is sensitive to the degree of symmetry in the energy deposition, allowing to distinguish QCD jets from quarks and gluon from boosted heavy particle decays. Compared to theoretical expectations, data show more spherical jets (see Fig. 6). The planar flow was also measured by the CDF collaboration as shown in Fig. 7 and it allows to measure the shape of the event. The planar flow vanishes for linear shape whereas it approaches unity for isotropic events. There is a reasonable agreement with QCD but data prefer more aplanar configurations than QCD.

\section{References}

[1] V. M. Abazov et al., Phys. Rev. Lett. 101 (2008) 062001; A. Abulencia et al., Phys. Rev. D 75, 092006 (2007); Phys. Rev. D 74, 071103 (2006).

[2] W.K. Tung et al., JHEP 0702, 053 (2007); J. Pumplin et al., JHEP 0207, 12 (2002); D. Stump et al., JHEP 0310, 046 (2003); A.D. Martin et al., Phys. Lett. B 604, 61 (2004).

[3] A.D. Martin, W.J. Stirling, R.S. Thorne, G. Watt, Eur. Phys. J. C 63189 (2009).

[4] V. M. Abazov et al., D0 Coll., Phys. Rev. D 80, 111107 (2009).

[5] V. M. Abazov et al., D0 Coll.,Phys. Lett. B693, 531 (2010).

[6] T. Aaltonen et al., CDF Coll., Phys. Rev. D 80, 111107 (2009).

[7] V. M. Abazov et al., D0 Coll., Phys. Rev. Lett. 103, 191803 (2009).

[8] D0 Coll., D0 Note 6043-CONF.

[9] T. Gleisberg et al., JHEP 0902007 (2009).

[10] T. Sjöstrand et al., Comp. Phys. Comm. 135, 238 (2001).

[11] D0 Coll., D0 Note 6032-CONF.

[12] CDF Coll., CDF note CDF/PUB/JET/PUBLIC/10199. 